\title{
Revisão do uso medicinal de Rhaphiodon echinus Schauer (Lamiaceae): Atividades biológica e farmacológica
}

\author{
Review of the medicinal use of Rhaphiodon echinus Schauer (Lamiaceae): biological and \\ pharmacological activities \\ Revisión del uso medicinal de Rhaphiodon echinus Schauer (Lamiaceae): Actividades biológicas y
}

farmacológicas

Marcos Aurélio Figueiredo dos Santos ORCID: https://orcid.org/0000-0002-3409-5242 Universidade Regional do Cariri, Brasil

E-mail: marcos.figueiredo@urca.br

Joice Layanne Guimarães Rodrigues ORCID: https://orcid.org/0000-0002-0683-5602 Universidade Regional do Cariri, Brasil

E-mail: joicelayanne17@gmail.com

Pedro Hudson Rodrigues Teixeira ORCID: https://orcid.org/0000-0001-5909-7642 Universidade Regional do Cariri, Brasil E-mail: pedrohudson@yahoo.com.br João Paulo Camilo de Oliveira ORCID: https://orcid.org/0000-0003-0286-1149 Universidade Regional do Cariri, Brasil E-mail: camilodeoliveirajoaopaulo35@gmail.com Dennis Bezerra Correia

ORCID: https://orcid.org/0000-0002-7782-4767 Universidade Regional do Cariri, Brasil E-mail: denniscorreia40@gmail.com

Saulo Almeida de Menezes

ORCID: https://orcid.org/0000-0001-6657-585X

Universidade Federal de Pernambuco, Brasil E-mail: saulomenezes99@gmail.com

Luciano Temoteo dos Santos

ORCID: https://orcid.org/0000-0002-9215-6832 Universidade Federal do Cariri, Brasil

E-mail: luciano.temoteosantos@gmail.com

Maraiza Gregorio de Oliveira

ORCID: https://orcid.org/0000-0002-2855-641X

Universidade Regional do Cariri, Brasil E-mail: maraaiza0104@ hotmail.com 


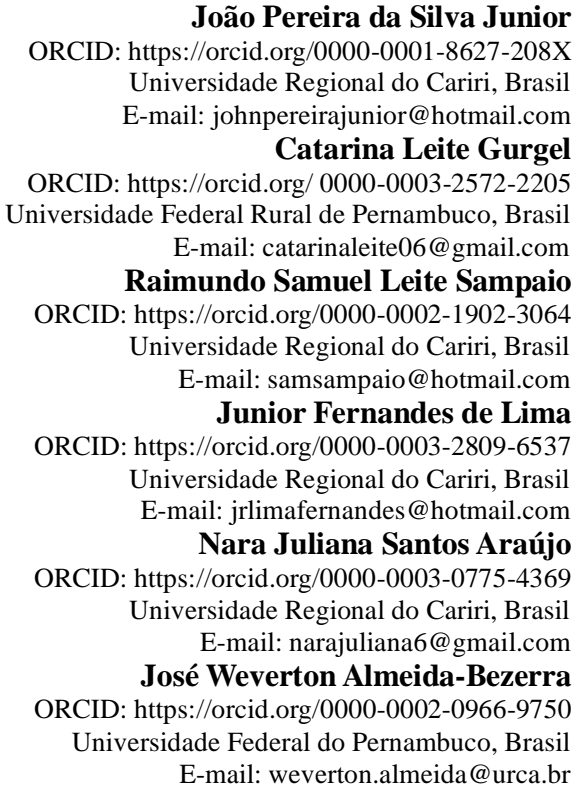

João Pereira da Silva Junior

ORCID: https://orcid.org/0000-0001-8627-208X Universidade Regional do Cariri, Brasil E-mail: johnpereirajunior@hotmail.com

Catarina Leite Gurgel

ORCID: https://orcid.org/ 0000-0003-2572-2205 Universidade Federal Rural de Pernambuco, Brasil

E-mail: catarinaleite06@gmail.com

Raimundo Samuel Leite Sampaio

ORCID: https://orcid.org/0000-0002-1902-3064 Universidade Regional do Cariri, Brasil E-mail: samsampaio@hotmail.com

Junior Fernandes de Lima

ORCID: https://orcid.org/0000-0003-2809-6537 Universidade Regional do Cariri, Brasil E-mail: jrlimafernandes@hotmail.com

Nara Juliana Santos Araújo

ORCID: https://orcid.org/0000-0003-0775-4369 Universidade Regional do Cariri, Brasil E-mail: narajuliana6@gmail.com

José Weverton Almeida-Bezerra

ORCID: https://orcid.org/0000-0002-0966-9750

Universidade Federal do Pernambuco, Brasil

E-mail: weverton.almeida@urca.br

\begin{abstract}
Resumo
Objetivou-se realizar uma revisão bibliográfica acerca das atividades biológica e farmacológica de Rhaphiodon echinus. Trata-se de uma revisão integrativa norteada pela seguinte pergunta: Quais as evidências cientificas acerca da atividade biológica e farmacológica da R. echinus? Elegeu-se as bases: MEDLINE, PUBMED, BDENF, SCOPUS e WoS para a pesquisa. Foi identificada uma população de 54 estudos cuja amostra caracterizou-se por nove artigos. Suscita-se que óleo essencial e os extratos etanólico e aquoso de R. echinus possuem compostos químicos de relevância em testes fitoquímicos e farmacológicos, demonstrando atividades antioxidantes e anti-inflamatória, além de atividade antimicrobiana contra Escherichia coli, Staphylococcus aureus e Pseudomonas aeruginosa ao potencializar a ação de fármacos como gentamicina e ciprofloxacino, assim como atividade antifúngica contra Candida albicans. Ademais, a atividade fitoterápica de R. echinus em chás, xaropes e banhos é observada contra inflamação uterina, gripe, cólica intestinal e infecção do trato urinário. Em suma, ressalta-se que são necessários novos estudos envolvendo $R$. echinus na área de etnobotânica a fim de melhor entender sua utilização e interfaces com o saber popular com vistas ao seu uso na prática clínica.
\end{abstract}

Palavras-chave: Sinergismo farmacológico; Plantas medicinais; Toxicologia; Medicamentos fitoterápicos.

\begin{abstract}
The objective was to conduct a literature review on the biological and pharmacological activities of Rhaphiodon echinus. This is an integrative review guided by the following question: What is the scientific evidence about the biological and pharmacological activity of $R$. echinus? The databases MEDLINE, PUBMED, BDENF, SCOPUS and WoS were chosen for the search. A population of 54 studies was identified whose sample was characterized by nine articles. It is suspected that the essential oil and the ethanolic and aqueous extracts of $R$. echinus have chemical compounds of relevance in phytochemical and pharmacological tests, showing antioxidant and anti-inflammatory activities, besides antimicrobial activity against Escherichia coli, Staphylococcus aureus and Pseudomonas aeruginosa by potentiating the action of drugs such as gentamicin and ciprofloxacin, as well as antifungal activity against Candida albicans. Furthermore, the phytotherapeutic activity of $R$. echinus in teas, syrups and baths is observed against uterine inflammation, influenza, intestinal colic and urinary tract infection. In summary, it is emphasized that new studies involving $R$. echinus are needed in the area of ethnobotany in order to better understand its use and interfaces with popular knowledge with a view to its use in clinical practice.
\end{abstract}

Keywords: Drug synergism; Plants medicinal; Toxicology; Phytotherapeutic drugs.

\title{
Resumen
}

El objetivo era realizar una revisión bibliográfica sobre las actividades biológicas y farmacológicas de Rhaphiodon echinus. Se trata de una revisión integradora guiada por la siguiente pregunta: ¿Cuáles son las pruebas científicas sobre la actividad biológica y farmacológica de $R$. echinus? Para la investigación se eligieron las bases de datos MEDLINE, PUBMED, BDENF, SCOPUS y WoS. Se identificó una población de 54 estudios cuya muestra se caracterizó por nueve artículos. Se sospecha que el aceite esencial y los extractos 
etanólicos y acuosos de $R$. echinus poseen compuestos químicos de relevancia en pruebas fitoquímicas y farmacológicas, demostrando una actividad antioxidante y antiinflamatoria, además de una actividad antimicrobiana contra Escherichia coli, Staphylococcus aureus y Pseudomonas aeruginosa a fin de potencializar la acción de fármacos como la gentamicina y el ciprofloxacino, así como una actividad antifúngica contra Candida albicans. Además, la actividad fitoterapéutica de $R$. echinus en tés, jarabes y baños se observa contra la inflamación uterina, la gripe, el cólico intestinal y la infección del tracto urinario. En resumen, se subraya que son necesarios nuevos estudios sobre $R$. echinus en el ámbito de la etnobotánica para comprender mejor su uso y las interfaces con el conocimiento popular con vistas a su utilización en la práctica clínica.

Palabras clave: Sinergismo farmacológico; Plantas medicinales; Toxicología; Fármacos fitoterapéuticos.

\section{Introdução}

Nos últimos anos, tem sido realizados estudos sobre a atividade farmacológica e biológica de plantas, especialmente aquelas com propriedades medicinais, com o objetivo de entender como funcionam seus princípios ativos com vistas ao uso tradicional nas comunidades e medicamentoso nas indústrias farmacêuticas para tratar doenças e, assim, prevenir agravos à saúde (Salkovic-Petrisic et al., 2015; Bueno, Martinez, \& Bueno, 2016; Ribeiro et al., 2014). Nesse sentido, há uma busca crescente por respostas específicas ao funcionamento bioquímico de certas partes da planta, como caule, folhas, raiz e frutos, ou em sua totalidade, como é o caso das plantas medicinais (Marinho, Silva, \& Andrade, 2011; Oliveira, Batista, Fernandes, Sales, \& Nogueira, 2016).

As plantas medicinais integram um conjunto de saberes dado por gerações e que são fontes independentes ligadas aos bens e serviços, seja do meio popular ou da atenção primária em saúde. De acordo com Viana, Souza e Nobre (2020), as plantas medicinais são importantes fontes de substâncias xenobióticas que podem ser utilizadas para a melhoria das condições de saúde do indivíduo, apresentando-se como tratamento eficaz para cura de certas doenças. De fato, alguns fitoterápicos compõem a política nacional de práticas integrativas e complementares ligadas à relação nacional de medicamentos disponível pelo sistema único de saúde (SUS), visando à busca por melhorias nas condições de saúde do indivíduo (Mattos, Camargo, Sousa, \& Zeni, 2018).

A utilização de plantas medicinais para tais fins ocorre há muito tempo pela civilização humana e, atualmente, é uma atividade impulsionada pela Organização Mundial da Saúde (OMS), tendo como princípio basilar o desenvolvimento de fitoterápicos capazes de agregar de modo significativo ao tratamento dos diferentes agravos em saúde (Alves, 2013; Viana, Souza, \& Nobre, 2020). Nessa perspectiva, o Brasil destaca-se por adotar resoluções que integram os fitoterápicos à atenção primária em saúde, bem como por normatizar a relação de plantas medicinais de interesse do Sistema Único de Saúde (SUS) (RENISUS) (Brasil, 2006; Antônio, Tesser, \& Moretti-Pires, 2014).

No entanto, um problema considerável na saúde pública decorre do uso indiscriminado de fitoterápicos que fazem interações medicamentosas com outros fármacos e acabam inativando, potencializando ou mesmo provocando reações indesejadas ao organismo, o que pode acarretar no agravo de doenças e trazer consigo danos irreparáveis (Oliveira, \& Lucena, 2015; Macêdo, Silva, Marcelino, \& Monte, 2020). Além disso, quando em doses não terapêuticas, o uso de plantas medicinais, principalmente através dos chás (método de infusão tradicional), leva ao desencadeamento de mecanismos de toxicidade subjacentes em vários tecidos corporais (Almeida, \& Albuquerque, 2002; Cunha, Silva, \& Roque, 2013; Virgínio et al., 2018).

Por outro lado, a atividade farmacológica de muitas plantas medicinais e de alguns produtos tradicionais já foi testada, inclusive com diversos princípios ativos isolados e identificados quimicamente (Silva, \& Souza, 2007; Rodrigues, \& Carvalho, 2011; Ribeiro et al., 2014), aprovados em âmbito nacional com prescrição, uso e fórmula, sendo 13 regulamentados no Brasil (Brasil, 2020). Por isso, encontrar novas plantas que viabilizem o cuidado com a saúde, desde que testadas, é um compromisso 
de todos os pesquisadores nas áreas de Química, Biologia e Farmacologia e áreas afins, fazendo-se necessária a participação da equipe multiprofissional em saúde junto à comunidade em geral para as devidas orientações.

De fato, diferentes levantamentos etnobotânicos relatam que variadas espécies de plantas, muitas das quais endêmicas da Caatinga, têm potencial para serem empregadas como fitofarmacológicos ativos no tratamento de dores, inflamações e outros distúrbios dos sistemas circulatório, respiratório e digestório (Alviano et al., 2008; Cartaxo, Souza, \& Albuquerque, 2010; Barbalho et al., 2011; Ribeiro et al., 2014; Macêdo et al., 2020; Viana, Souza, \& Nobre, 2020). Neste contexto, destacase a espécie Rhaphiodon echinus Schauer, representante da família botânica Lamiaceae e conhecida popularmente como "betonica" ou "bentônica", endêmica da caatinga e, portanto, localizada na Região Nordeste do Brasil, especialmente nos estados com regiões litorâneas (Pio et al., 2019). Rhaphiodon echinus está adaptada à região dos sertões e semiárido brasileiros, uma particularidade fitoclimática importante para o crescimento e desenvolvimento desta espécie (Viana, Souza, \& Nobre, 2020).

O conhecimento popular tem-se apropriado, ao longo dos anos, do uso dessa e de outras plantas para fins medicinais e convergem com os ensinamentos de profissionais para o tratamento de diferentes afecções como tosse, fadiga, dores, infecções e inflamações em geral. Estudos relatam que a espécie Rhaphiodon echinus também pode ser promissora no tratamento de cólicas intestinais, gripe e até hipertensão arterial sistêmica (Mattos et al., 2018; Pio et al., 2019). Diante do exposto, este estudo tem como objetivo mapear as evidências científicas acerca da atividade biológica e farmacológica da espécie R. echinus Schauer, a fim de suscitar as principais informações que viabilizem seu uso como fitoterápico.

\section{Metodologia}

Este estudo configura-se como uma revisão integrativa da literatura, de caráter exploratório e investigativo, que permite agregar diferentes informações para a formulação de conclusões gerais a respeito de determinada área do conhecimento, mediante uma síntese de estudos publicados na perspectiva em análise.

Este trabalho compreendeu as seguintes etapas: I. Identificação do tema e seleção da hipótese ou questão de pesquisa para a elaboração da revisão integrativa; II. Estabelecimento de critérios para inclusão e exclusão de estudos/amostragem ou busca na literatura; III. Definição das informações a serem extraídas dos estudos selecionados/categorização dos estudos; IV. Avaliação dos estudos incluídos na revisão integrativa; V. Interpretação dos resultados; e VI. Apresentação da revisão/síntese do conhecimento. Esta metodologia de estudo baseou-se em estudos realizados previamente por Mendes, Silveira e Galvão (2019).

A questão norteadora da presente revisão foi: Quais as evidências cientificas acerca da atividade biológica e farmacológica da Rhaphiodon echinus? As bases elegíveis para a realização desta pesquisa foram: Medical Literature Analysis and Retrieval System Online (MEDLINE) via EBSCO Information Services, National Library of Medicine National Institutes of Health (PUBMED), SCOPUS Preview, Web of Science, além de Scientific Electronic Library Online e Science Direct, utilizando o método de busca avançada e a categoria título, resumo e palavras-chaves.

Em cada base de dados, os descritores de assunto do Medical Subject Heading (MeSH) da PubMed foram delimitados e cruzados, sendo utilizados: Rhaphiodon echinus Schauer, Rhaphiodon echinus, Hyptis sideritis (sinônimo de R.echinus) e seus DeCS, com os operadores booleanos AND e OR, de forma pareada por pesquisadores distintos. Os artigos foram então submetidos a um processo de filtragem constituído pelos critérios de inclusão: artigos disponíveis eletronicamente com texto completo online; em horizonte temporal, classificados como artigos originais; estudos primários, publicados em português, inglês ou espanhol e que atendem à pergunta norteadora. Foram excluídos os estudos duplicados e repetidos e os que não 
abordassem a temática investigada (Figura 1). A abrangência temporal de publicação incluiu trabalhos publicados nas duas últimas décadas.

Para coleta de dados, utilizou-se o formulário adaptado que organiza as principais informações referentes aos estudos (Autor/ Parte da planta/ Método/ Organismo/ Atividade), assim como dados do desenvolvimento do artigo (Ursi, \& Gavão, 2006). Além disso, foi utilizado o instrumento Preferred Reporting Items for Systematic Rewiew and Meta-Analyses (PRISMA) para expor a busca dos estudos (Fuchs, \& Paim, 2010) (Figura 1).

Figura 1. Fluxograma do processo de busca e seleção dos artigos.

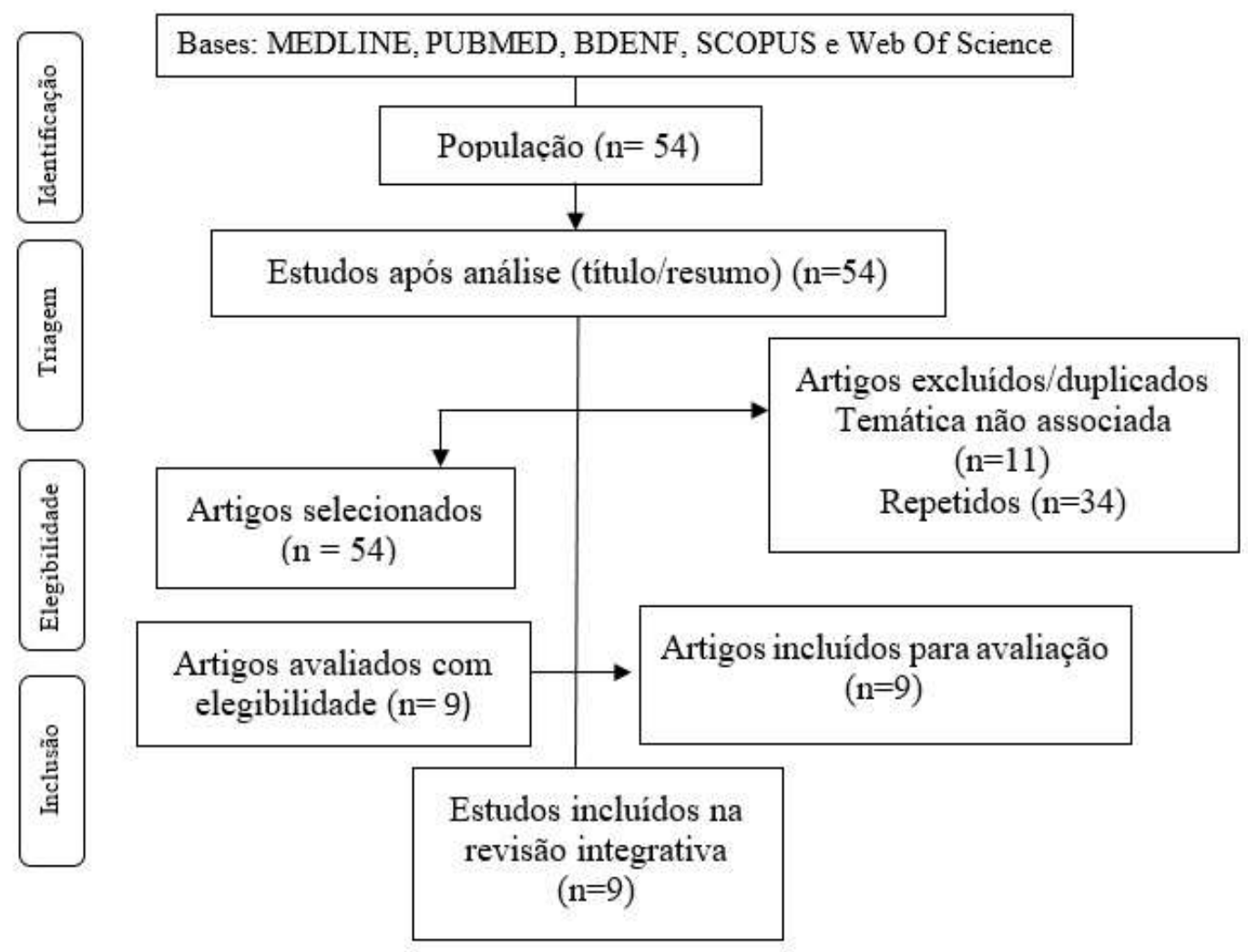

Fonte: Autores (2020).

\section{Resultados}

Com base nos passos metodológicos acima descritos, foi possível trazer uma abordagem geral sobre os efeitos biológicos e farmacológicos de Rhaphiodon echinus (Schauer), assim como sobre seu uso popular, destacando as principais informações que viabilizem seu uso como fitoterápico promissor no tratamento de diversas afecções (Quadro 1). 
Quadro 1. Atividades biológica e farmacológica de componentes fitoquímicos da espécie R. echinus.

\begin{tabular}{|c|c|c|}
\hline $\begin{array}{l}\text { Autor/ } \\
\text { Parte testada }\end{array}$ & Metodologia & Compostos identificados / Resultados \\
\hline $\begin{array}{l}\text { Duarte et al., } \\
2016 \mathrm{a} \\
\text { Óleo essencial }\end{array}$ & $\begin{array}{l}\mathrm{O} \text { óleo foi extraído da planta seca e } \\
\text { submetido à hidrodestilação em um } \\
\text { aparelho Clevenger. Utilizaram-se } \\
\text { bactérias na testagem. }\end{array}$ & $\begin{array}{l}\text { a) O óleo essencial mostrou relação positiva na ação contra } \\
\text { Candida krusei, Candida tropicalis e Pseudomonas } \\
\text { aeruginosa; b) O óleo essencial inativou o ferro }\left(\mathrm{Fe}^{2+}\right) \text { na } \\
\text { reação química com o ácido ascórbico (vitamina } \mathrm{C}) \text {; c) O } \\
\text { efeito combinado do óleo essencial e do fluconazol não } \\
\text { teve efetividade contra Candida albicans; d) A união entre } \\
\text { óleo essencial e amicanica demostrou inibição contra } \\
\text { Escherichia coli; e) Antibióticos como a gentamicina, } \\
\text { amicacina, imipenem e ciprofloxacino não tiveram } \\
\text { atividade significativa, quando combinados ao óleo } \\
\text { essencial, contra Staphylococcus aureus; f) A Rhaphiodon } \\
\text { echinus apresentou antagonismo em relação à nistatina. }\end{array}$ \\
\hline $\begin{array}{l}\text { Duarte et al., } \\
2016 b\end{array}$ & $\begin{array}{l}\text { Foi realizada a determinação da } \\
\text { quantidade total de fenólicos e ação } \\
\text { antioxidante. Verificou-se a inativação } \\
\text { para o ferro. Determinação da } \\
\text { fragilidade osmótica para eritrócitos, } \\
\text { mostrando genotoxicidade. Nos testes } \\
\text { foram empregados: Ácido Gálico; } \\
\text { Ácido Clorogênico; Ácido Caféico; } \\
\text { Ácido Elágico; Quercitrina; Rutina e } \\
\text { Quercetina. }\end{array}$ & $\begin{array}{l}\text { a) A quercetina e a quercitrina são compostos fitoquímicos } \\
\text { detectados em grandes quantidades no extrato de } R \text {. } \\
\text { echinus; b) } \mathrm{O} \text { extrato aquoso e etanólico de } R \text {. echinus } \\
\text { exibiu atividade antioxidante; c) } \mathrm{O} \text { extrato aquoso e } \\
\text { etanólico exibiu efeito protetor contra } \mathrm{Fe}^{2+} \text { induzido por } \\
\text { peroxidação lipídica; d) Os extratos aquoso e etanólico não } \\
\text { apresentaram alterações visíveis nos ensaios citotóxicos } \\
\text { com leucócitos humanos; c) Não foi demonstrada diferença } \\
\text { significativa entre eritrócitos tratados e não tratados com os } \\
\text { extrato aquosos de } R \text {. echinus; d) Não foram encontradas } \\
\text { diferenças significativas no índice de dano ao DNA entre } \\
\text { leucócitos tratados e não tratados com o extrato aquoso do } \\
\text { R. echinus. }\end{array}$ \\
\hline Costa & $\begin{array}{l}\text { Extração do óleo essencial. Lixiviação } \\
\text { do radical livre do óleo essencial de R. } \\
\text { echinus. Teste de letalidade com } \\
\text { Artemia Salina. Nos testes } \\
\text { empregaram-se: Ácido Galico; Ácido } \\
\text { Caféico; Ácido Clorogênico; Rutina e } \\
\text { Quercetina. }\end{array}$ & $\begin{array}{l}\text { a) Os principais constituintes observados no período da } \\
\text { manhã foram o ácido cafeico, o ácido gálico e a quercetina; } \\
\text { b) No período da tarde, quercetina e ácido caféico; c) O } \\
\text { óleo essencial apresentou moderada ação antioxidante, } \\
\text { comparado ao ácido ascórbico; d) Os ensaios toxicológicos } \\
\text { com Artemia salina mostraram toxicidade significativa. }\end{array}$ \\
\hline Costa et al., 2017 & $\begin{array}{l}\text { Preparação do extrato aquoso e } \\
\text { etanólico de } R \text {. echinus. Efeito } \\
\text { modulatório e extrato de } R \text {. echinus. } \\
\text { Toxicidade e teste com A. salina. Nos } \\
\text { testes empregaram-se: Testagem com } \\
\text { Metanol, Ácido Acético, Ácido } \\
\text { Gálico, Ácido Caféico, Ácido Elágico } \\
\text { e Ácido Clorogênico. Foram utilizados } \\
\text { também Quercetina, quercitrina, rutina }\end{array}$ & $\begin{array}{l}\text { a) Em análise à fitoquímica dos extratos aquoso e etanólico, } \\
\text { foi possível observar a presença de ácido gálico, ácido } \\
\text { clorogênico, ácido caféico, rutina e quercetina; b) Não foi } \\
\text { observada relevância clínica contra as espécies do gênero } \\
\text { Candida; c) A associação entre extrato aquoso e drogas } \\
\text { antifúngicas como a nistatina mostrou efeito antagônico } \\
\text { contra } C \text {. albicans e Candida tropicalis, quando comparado } \\
\text { ao controle; d) O extrato etanólico potencializou o efeito da } \\
\text { nistatina; e) Os extratos aquoso e etanólico mostraram }\end{array}$ \\
\hline
\end{tabular}




\begin{tabular}{|c|c|c|}
\hline & e luteolina. & $\begin{array}{l}\text { atividade antibactericida quando comparados com os } \\
\text { fármacos-controle gentamicina e amicacina, } \\
\text { respectivamente, para Escherichia coli; f) O extrato aquoso } \\
\text { aumentou significativamente a atividade antibiótica de } \\
\text { gentamicina e imipenen; g) O extrato etanólico possuiu } \\
\text { atividade significativa para Pseudomonas aureginosa } \\
\text { quando comparada aos fármacos gentamicina, imipenen, } \\
\text { amicacina e ciprofloxacino; h) Nenhum dos extratos } \\
\text { apresentou efeito significativo para } S \text {.aureus. }\end{array}$ \\
\hline Pio et al., 2019 & $\begin{array}{l}\text { Utilização de dados etnobotânicos com } \\
\text { fitoquímica preliminar e análise } \\
\text { farmacológica de } R \text {. echinus. Emprego } \\
\text { de testes farmacológicos. Utilizaram- } \\
\text { se bactérias nos testes. }\end{array}$ & $\begin{array}{l}\text { a) Os extratos apresentaram atividade antibacteriana para: } \\
\text { Pseudomonas aeruginosa, S. aureus, E. Coli; b) } \\
\text { Demonstraram relaxamento de } 80 \% \text { do musculo uterino; c) } \\
\text { A fração acetato de etila e etanólico, apresentou forte } \\
\text { potencial antibacteriano sobre as cepas testadas. }\end{array}$ \\
\hline $\begin{array}{l}\text { Ferreira et al, } \\
2019\end{array}$ & $\begin{array}{l}\text { Emulsão dos extratos. Preparo das } \\
\text { espécies fúngicas. Meio de cultura. } \\
\text { Utilização do fármaco antifúngico } \\
\text { nistatina em pó. Determinação da } \\
\text { concentração inibitória mínima. } \\
\text { Fungos foram utilizados nos testes }\end{array}$ & $\begin{array}{l}\text { a) A Concentração inibitória teve forte atividade } \\
\text { antifúngica; b) A menor concentração do extrato aquoso de } \\
\text { R. echinus foi de } 256 \mu \mathrm{g} / \mathrm{mL} \text { para as cepas bacterianas. }\end{array}$ \\
\hline
\end{tabular}

Fonte: Autores (2020).

Em estudos de levantamentos etnobotânicos, é possível observar a integração de uma gama de saberes populares do uso medicinal da espécie R. echinus (Schauer), incluindo as partes da planta utilizada e o seu modo de preparo (Quadro 2).

Quadro 2. Uso etnobotânico de R. echinus.

\begin{tabular}{|l|l|l|l|}
\hline Referências & Utilidade & Parte usada & Apresentação/ preparo/ nome vernacular \\
\hline Albuquerque et al., 2007 & Inflamação Uterina & Folha, Raiz & $\begin{array}{l}\text { Erva } \\
\text { Flor de Urubu }\end{array}$ \\
\hline $\begin{array}{l}\text { Moreira e Bragança, } \\
2011\end{array}$ & $\begin{array}{l}\text { Anti-inflamatória } \\
\text { atividade analgésica }\end{array}$ & $\begin{array}{l}\text { Extrato } \\
\text { aplicado em feridas }\end{array}$ & - \\
\hline Brandão et al., 2012 & $\begin{array}{l}\text { Gripe e cólica intestinal; } \\
\text { Infecção do trato urinário; } \\
\text { Dismenorreia; } \\
\text { Inflamação dental Tosse; }\end{array}$ & Folha & $\begin{array}{l}\text { Folha } \\
\text { Chá, Xarope, Banho } \\
\text { Betônica, flor de urubu, falsa menta, menta } \\
\text { rasteira. }\end{array}$ \\
\hline Duarte et al.. 2016a & $\begin{array}{l}\text { Efeito antimicrobiano } \\
\text { contra E. coli. }\end{array}$ & Óleo & - \\
\hline
\end{tabular}

Fonte: Autores (2020). 
O quadro permite aventar que as indicações terapêuticas perpassam os saberes populares e estão em consonância aos saberes científicos cunhados ao longo de anos pelos estudiosos da área, demonstrando, através de experimentos científicos laboratoriais, os efeitos antioxidantes e anti-inflamatórios de $R$. ecinhus, assim como sua ação antifúngica no tratamento de candidíase oral e recuperação de distúrbios do trato digestivo superior. Na Figura 2 pode ser observada uma imagem da Rhaphiodon echinus.

Figura 2. Imagem da Rhaphiodon echinus na natureza.

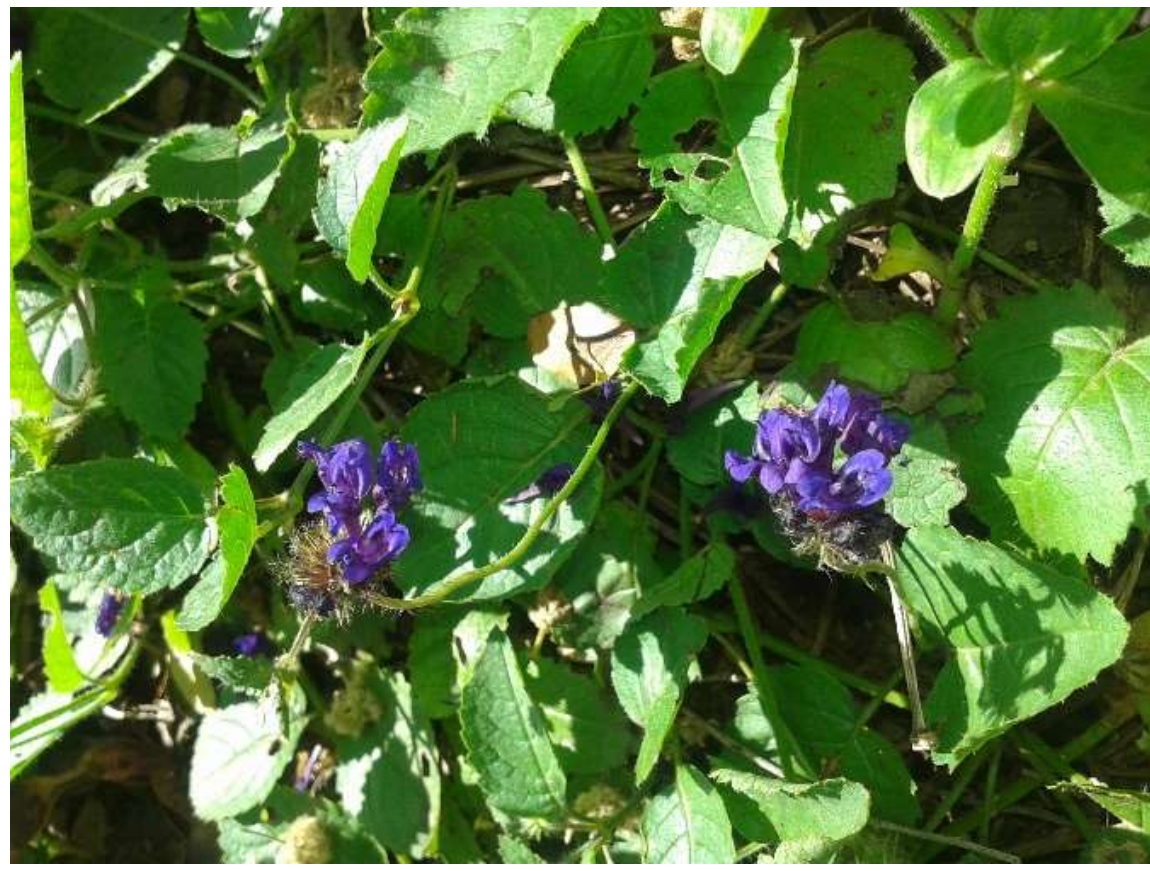

Fonte: Autores (2020).

\section{Discussão}

Rhaphiodon echinus é uma espécie de planta nativa da caatinga conhecida por apresentar um potencial uso terapêutico já introduzido no meio popular e demonstrado através de testes laboratoriais específicos envolvendo seu óleo essencial e extrato das folhas, além de avaliação do efeito protetor dos constituintes majoritários de $R$. echinus contra microrganismos patogênicos (Moreira, \& Bragança, 2011; Ferreira et al., 2019). As reações entre os componentes fitoquímicos da espécie $R$. echinus foram mais bem analisadas com seu óleo essencial e extrato aquoso, demostrando efeito antioxidante, antibactericida e antifúngico (Costa et al., 2017; Pio et al., 2019).

Testes conduzidos com extratos de $R$. echinus exibiram uma atividade imunomodulatória promissora em relação à ação antifúngica para tratamento da candidíase oral e do trato digestivo superior (Ferreira et al., 2019). A atividade modulatória do extrato demostrou significância quando combinada aos padrões antibacterianos: gentamicina, amaicacina, ciprofloxacino e imipenen (Costa et al., 2017). Por outro lado, o óleo essencial de R. echinus não apresentou atividade promissora na inativação de Candida albicans e Staphylococcus aureus, potencializando apenas a ação de Candida Krusei e Candida tropicalis, mas houve inibição da atividade de Escherichia coli e atividade protetora do óleo essencial contra Pseudomonas aeruginosa (Duarte et al., 2016a).

De fato, Oliveira et al (2016) ressaltaram que o óleo essencial de muitas plantas medicinais, incluindo $R$. ecinhus, tem ação relevante contra a candidíase, demostrando baixa toxicidade. Além disso, o óleo essencial de $R$. echinus é relatado por ter 
importante uso no tratamento da gripe e cólicas intestinais (Pio et al., 2019) e exibir atividade antioxidante ao demonstrar efeito quelante de íons ferro $\left(\mathrm{Fe}^{2+}\right)$ quando comparado ao ácido ascórbico (Duarte et al., 2016a).

Estudos realizados com elementos figurados do sistema sanguíneo analisaram os potenciais efeitos de $R$. echinus no tratamento de infecções e doenças que afetam esse sistema (Duarte et al., 2016b). Verificou-se que o extrato dessa planta não produziu alterações nos leucócitos humanos, assim como nos eritrócitos, demonstrando a baixa citotoxicidade de R. echinus (Duarte et al., 2016b).

Acredita-se que quanto mais o saber científico é imbricado na sociedade, mais o uso dessa e de outras plantas medicinais pode ser alvo de novas perspectivas terapêuticas, desde que testadas e aprovadas. Em estudo que avaliou o conhecimento do uso de plantas medicinais entre profissionais de saúde da Estratégia Saúde da Família, 96,2\% dos profissionais acreditam no poder medicinal curativo das plantas (Mattos et al., 2018). A utilização de plantas medicinais na Estratégia Saúde da Família reflete a relação entre o processo saúde-doença e a facilidade de cultivo e preparação das ervas (Zeni et al., 2017).

A isso, os marcadores concentração/composição são apresentados em normativas nacionais desde 2014 com a manipulação pelo SUS, farmácias vivas e até farmácias conveniadas, assegurando o potencial terapêutico das 13 plantas listadas pela RENAME (Brasil, 2020). Esse conhecimento sobre o uso de plantas medicinais perpassa as comunidades a partir dos saberes indígenas advindos de pais ou mães que vivenciaram essas experiências (Castro, \& Figueiredo, 2019). Nesses casos, a preferência por produtos naturais, sejam chás, xaropes ou banhos de partes do vegetal, é de extrema importância no melhor cuidado com a saúde em detrimento das fórmulas industrializadas (Cruz et al. 2017; Pio et al., 2019).

Em estudos etnobotânicos, os chás representam o modo de preparo mais citado para as plantas medicinais (Marinho, Silva, \& Andrade, 2011) e, de acordo com Viana, Souza e Nobre (2020), o uso da casca em banhos é frequentemente citado em outros estudos etnobotânicos do semiárido nordestino como sendo a segunda parte vegetal mais utilizada (Lucena et al., 2008; Lucena et al., 2011; Ribeiro et al., 2014). Com destaque para $R$. echinus, suas partes mais utilizadas são as folhas e as raízes, sendo preparadas geralmente em chás, xaropes e banhos. Sua indicação como fitoterapia caseira destaca-se contra inflamações em geral, principalmente inflamações uterinas, frescor em feridas, cólica intestinal, infecção do trato urinário, dismenorreia e tosse. Isso é justificável em virtude de $R$. echinus apresentar atividade anti-inflamatória e analgésica, assim como efeito antimicrobiano contra E.coli, conforme mencionado previamente (Moreira, \& Bragança, 2011; Costa et al., 2017).

Além de $R$. echinus, os fitoterápicos mais conhecidos por profissionais de saúde são a Matricaria chamomilla (Camomila-vulgar), Melissa officinalis (erva-cidreira), Peumus boldus (boldo do Chile) e Passiflora edulis (maracujá) (Mattos et al., 2018). Soma-se a isso plantas medicinais de alta versatilidade por apresentarem efeitos antioxidantes e antibacterianos, entre as quais destacam-se Mentha spicata (Hortelã), Dysphania ambrosioides (Mastruz), Aloe vera (babosa) e Anethum graveolens (Endro) (Viana, Souza, \& Nobre, 2020; Haluch et al., 2020).

No entanto, muitas plantas medicinais ainda são desconhecidas por profissionais de saúde e por isso não prescritas, o que causa dificuldade de adesão na prática e influencia diretamente na vida dos pacientes (Andrade et al., 2017). Isso se dá principalmente pela preferência a medicamentos fitoterápicos e a formação biomédica predominante ao demostrar um censo cético quanto ao uso da fitoterapia da comunidade. Por isso, o Formulário Técnico Nacional (FTN) e os Protocolos Clínicos e Diretrizes Terapêuticas (PCDT) foram normatizados (Antônio, Tesser, \& Moretti-Pires, 2014; Brasil, 2020) e uma classificação especifica no que diz respeito às plantas medicinais foi disponibilizada pela OMS, chamada de Herbal ATC Index, que prioriza a planta de acordo com o grupo anatômico principal delineando o seu uso (Brasil, 2020). 


\section{Conclusão}

Os estudos apontam a espécie $R$. echinus como uma importante planta para a inibição de crescimento de bactérias e fungos, apresentando resultados relevantes contra Candida albicans e Staphylococcus aureus, além de potencializar a ação de fármacos de primeira linha como gentamicina, amaicacina, ciprofloxacino e imipenen.

Os óleos essenciais e os extratos vegetais são mais bem relatados nos estudos com Rhaphiodon echinus por possuírem compostos químicos de relevância veiculados a testes farmacológicos, fitoquímicos e imunomodulatórios. No entanto, ainda há uma lacuna existente na literatura quanto aos mecanismos elucidatórios da ação de $R$. echinus frente aos elementos sanguíneos e sua possível toxicidade.

No campo da saúde, Rhaphiodon echinus revela atividade fitoterápica no saber popular relacionado ao seu uso em chás, xaropes e banhos. Neste sentido, a referida planta é indicada contra inflamação uterina, gripe, cólica intestinal, infecção do trato urinário, dismenorreia e tosse devido à sua atividade anti-inflamatória e analgésica, além de atividade antimicrobiana contra Escherichia coli e Pseudomonas aeruginosa.

Por fim, ressalta-se que são necessários novos estudos na área de etnobotânica em relação às atividades farmacológicas e biológicas de Rhaphiodon echinus a fim de melhor entender a utilização desta planta medicinal e suas interfaces com o saber popular com vistas ao seu uso efetivo na prática clínica.

\section{Referências}

Almeida, C. F. C. B.R., \& Albuquerque, U.P. (2002). Uso e conservação de plantas e animais medicinais no estado de Pernambuco (Nordeste do Brasil): um estudo de caso. Interciencia, 27(6), 276-285.

Alves, L. F. (2013). Produção de fitoterápicos no Brasil: história, problemas e perspectivas. Revista Virtual de Química, 5(3), 450-513.

Alviano, W. S., Alviano, D. S., Diniz, C. G., Antoniolli, A. R., Alviano, C. S., Farias, L. M., Carvalho, M. A. R., Souza, M. M. G., \& Bolognese, A. M. (2008). In vitro antioxidant potential of medicinal plant extracts and their activities against oral bacteria based on Brazilian folk medicine. Archives of oral biology, 53(6), 545-552.

Andrade, S. A. L., Tristão, M. I. S., Miguel, M. D., Dias, J. D. F. G., Gomes, E. C., Burci, L. M., \& Paula, C. S. (2017). Fitoterápicos da relação nacional de medicamentos essenciais no Brasil. Revista Cubana de Plantas Medicinales, 22(1), 1-18.

Antônio, G. D., Tesser, C. D., \& Moretti-Pires, R. O. (2014). Phytotherapy in primary health care. Revista Saúde Pública, 48(3), $541-553$.

Barbalho, S. M., Farinazzi, F. M. V., Guiguer, E. L., Silva, P. H., Silva, V. S., Oshiiwa, M., \& Goulart, R. A. (2011). Espécies de Mentha Podem Auxiliar na Redução de Fatores de Risco Vascular em Pacientes Diabéticos. Saúde e Pesquisa, 4(3), 387-392.

Brasil. (2009). Ministério da Saúde RENISUS. Relação nacional de plantas medicinais de interesse ao SUS. Espécies vegetais. http://portal.saude.gov.br/portal/arquivos/pdfRENISUS.pdf.

Brasil. (2020). Ministério da Saúde. Secretaria de Ciência, Tecnologia, Inovação e Insumos Estratégicos em Saúde. Departamento de Assistência Farmacêutica e Insumos Estratégicos. Relação $\quad$ Nacional de http://bvsms.saude.gov.br/bvs/publicacoes/relacao_medicamentos_rename_2020.pdf.

Bueno, M. J. A., Martinez, B. B., \& Bueno, J. C. (2016). Manual de plantas medicinais e fitoterápicos utilizados na cicatrização de feridas. Universidade do Vale do Sapucaí - Univás Mestrado Profissional em Ciências Aplicadas à Saúde. Univás: Pouso Alegre.

Cartaxo, S. L., de Souza, M. M. A., \& Albuquerque, U. P. (2010). Medicinal plants with bioprospecting potential used in semi-arid northeastern Brazil. Journal of ethnopharmacology, 131(2), 326-342.

Castro, M. R., \& Figueiredo, F. F. (2019). Saberes tradicionais, biodiversidade, práticas integrativas e complementares: o uso de plantas medicinais no SUS. Hygeia-Revista Brasileira de Geografia Médica e da Saúde, 15(31), 56-70.

Costa, A. R., de Lima Silva, J., Lima, K. R. R., Rocha, M. I., Barros, L. M., da Costa, J. G. M., Boligon, A. A., Kamdem, J. P., Carneiro, J. N. P., Leite, N. F., Menezes, I. R. A., Duarte, A. E., Morais-Braga, M. F. B., \& Coutinho, H. D. M. (2017). Rhaphiodon echinus (Nees \& Mart.) Schauer: Chemical, toxicological activity and increased antibiotic activity of antifungal drug activity and antibacterial. Microbial pathogenesis, 107(1), 280-286.

Cruz, M. C. S., Santos, P. O., Barbosa Jr, A. M., Mélo, D. L. F. M., Alviano, C. S., Antoniolli, A. R., Alviano, D. S, \& Trindade, R. C. (2017). Antifungal activity of Brazilian medicinal plants involved in popular treatment of mycoses. Journal of ethnopharmacology, 111(2), 409-412.

Cunha, A. P., Silva, A. P., \& Roque, O. R. (2013). Plantas e produtos vegetais em fitoterapia. Lisboa: Fundação Calouste Gulbenkian. 
Duarte, A. E., Menezes, I. R. A., Morais-Braga, M. F. B., Leite, N. F., Barros, L. M., Waczuk, E. P., Silva, M. A. P., Boligon, A., Rocha, J. B. T., Souza, D. O., Kamdem, J. P., Coutinho, H. D. M., \& Burger, M. E. (2016a). Antimicrobial activity and Modulatory effect of essential oil from the leaf of Rhaphiodon echinus (Nees \& Mart) Schauer on some antimicrobial drugs. Molecules, 21(6), 1-14.

Duarte, A. E., Waczuk, E. P., Roversi, K., Silva, M. A. P., Barros, L. M., Cunha, F. A. B., Menezes, I. R. A., Costa, J. G. M., Boligon, A. A., Ademiluyi, A. O., Kamdem, J. P., Rocha, J. B. T., \& Burger, M. E. (2016b). Polyphenolic composition and evaluation of antioxidant activity, osmotic fragility and cytotoxic effects of Raphiodon echinus (Nees \& Mart.) Schauer. Molecules, 21(1), 1-15.

Ferreira, J. L. S., Cavalcante, J. N. M., Siqueira, D. S., Bezerra, R. V., Delgado, L. A., Oliveira, H. M. B. F., Vieira, E. S. S., Maia, G. L. A., Lima, E. O., \& Oliveira Filho, A. (2019). Comparação da atividade antifúngica do extrato aquoso e do extrato etanólico de Rhaphiodon echinus (Lamiaceae) contra cepas Candida tropicalis. Revista da Universidade Vale do Rio Verde, 17(1), 1-8.

Fuchs, S. C. P. C., \& Paim, B. S. (2010). Revisão sistemática de estudos observacionais com metanálise. Revista HCPA, 30(3) $294-301$.

Haluch, S. M., Schellin, L. M., Pan, V. X. X. C., de Oliveira Alves, A. L., dos Santos, M. C., Chemin, A. P., Lopes, V. E., \& Staichok, C. (2020). Prospecção de novos antimicrobianos e bactericidas frente a microrganismos de interesse de saúde pública. Brazilian Journal of Animal and Environmental Research, 3(4), 3630-3652.

Lucena, R. F. P., Nascimento, V. T., Araújo, E. L., \& Albuquerque, U. P. (2008). Local uses of native plants in an area of Caatinga vegetation (Pernambuco, NE Brazil). Ethnobotany Research and Applications, 6, 3-14.

Lucena, R. F. P., Farias, D. C., Carvalho, T. K. N., Lucena, C. M., Vasconcelos Neto, C. F. A., \& Albuquerque, U. P. (2011). Conhecimento tradicional de Myracrodruon Urundeuva Allemão por comunidades tradicionais no Semiárido do Nordeste do Brasil. Sitientibus série Ciências Biológicas, 2 , $255-264$.

Macêdo, L. P. V., Silva, J. R. L., Marcelino, E. M., \& Monte, N. L. (2020). Conhecimento e uso de plantas medicinais por idosos atendidos na atenção primária à saúde. Realize Editora, 6(1), 384-404

Marinho, M. G. V., Silva, C. C., \& Andrade, L.H.C. (2011). Levantamento etnobotânico de plantas medicinais em área de caating a no município de São José de Espinharas, Paraíba, Brasil. Revista Brasileira de Plantas Medicinais, 13(2), 170-182.

Mattos, G., Camargo, A., Sousa, C. A. D., \& Zeni, A. L. B. (2018). Plantas medicinais e fitoterápicos na Atenção Primária em Saúde: percepção dos profissionais. Ciência \& Saúde Coletiva, 23(11), 3735-3744.

Mendes, K.D.S., Silveira R.C.C.P., Galvão, C.M. (2019). Uso de gerenciador de referências bibliográficas na seleção dos estudos primários em revisão integrativa. Texto Contexto Enferm. 28; e20170204:1-13.

Oliveira, D. M. S, \& Lucena, E. M. P. (2015). O uso de plantas medicinais por moradores de Quixadá- Ceará. Revista Brasileira de Plantas Medicinais, Campinas, 17(3), 407-412.

Oliveira, L. B. S., Batista, A. H. M., Fernandes, F. C., Sales, G. W. P., \& Nogueira, N. A. P. (2016). Atividade antifúngica e possível mecanismo de ação do óleo essencial de folhas de Ocimum gratissimum (Linn.) sobre espécies de Candida. Revista Brasileira de Plantas Medicinais, 18(2), 511-523.

Pereira, L. C. O. (2014). Caracterização química de óleos essenciais de quatro espécies da família Lamiaceae: Hyptis suaveolens (L.) Poit, Hyptis pectinata (L.) Poit, Hyptis martiusii Benth. e Rhaphiodon echinus (Nees \& Mart.) Schauer (Trabalho de Conclusão de Curso). Graduação em Farmácia da Universidade Federal da Paraíba, João Pessoa, PB, Brasil.

Pio, I. D. S. L., Lavor, A. L., Damasceno, C. M. D., Menezes, P. M. N., Silva, F. S., \& Maia, G. L. A. (2019). Traditional knowledge and uses of medicinal plants by the inhabitants of the islands of the São Francisco river, Brazil and preliminary analysis of Rhaphiodon echinus (Lamiaceae). Brazilian Journal of Biology, 79(1), 87-99.

Ribeiro, D. A., Macêdo, D. G., Oliveira, L. G. S., Saraiva, M. E., Oliveira, S. F., Souza, M. M. A., \& Menezes, I. R. A. (20 14). Potencial terapêutico e uso de plantas medicinais em uma área de Caatinga no estado do Ceará, nordeste do Brasil. Revista Brasileira de Plantas Medicinais, 16(4), 912-930.

Rodrigues, C. A., Pereira, P. S., Sousa, M. K. A., Rodrigues, F. C., Mendes, V. R. D., Lima, R. K. R., Barros, L. M., Sousa, C. M. R., Alcantara, M. S., Cavalcante, A. B. L., Macedo, R. C., Kamdem, J. P., \& Duarte, A. (2018). Potencial atividade antioxidante e toxicológica do óleo essencial de Rhaphiodon echinus (Nees \& Mart) Schauer (Lamiaceae): morfoanatomia e composição polifenólica de seus extratos. Phyton, 87, 79-86.

Rodrigues, V. E. G., \& Carvalho, D. A. (2011). Levantamento etnobotânico de plantas medicinais do domínio cerrado na região do Alto Rio Grande, Minas Gerais. Ciência Agrotécnica, 25, 102- 123.

Salkovic-Petrisic, M., Knezovic, A., Osmanovic-Barilar, J., Smailovic, U., Trkulja, V., Riederer, P., Amit T., Mandel S., \& Youdim, M. B. (2015). Multitarget iron-chelators improve memory loss in a rat model of sporadic Alzheimer's disease. Life sciences, 136, $108-119$.

Silva, J. O., \& Souza, P. S. (2007). Levantamento etnobotânico das plantas medicinais utilizadas pela população da Vila Canaã região sudoeste - Goiânia, Goiás (Trabalho de Conclusão de Curso). Uni-Anhanguera, Centro Universitário de Goiás, Goiânia, GO, Brasil.

Ursi, E. S, \& Galvão, C. M. (2006). Prevenção De Lesões De Pele No Perioperatório: Revisão Integrativa da Literatura. Revista Latino-Am Enfermagem, 14(1), 124-131.

Viana, J. W. M., Souza, J. J., \& Nobre, F. W. (2020). Uso popular de plantas medicinais por moradores de uma comunidade rural em Crato-CE. Revista Interdisciplinar Encontro das Ciências, 3(3), 1513 - 1531.

Virgínio, T. B., Castro, K. S., Lima, A. L. A., Rocha, J. V., Bonfim, I. M., \& Campos, A. L. (2018). Utilização de plantas medicinais por pacientes hipertensos e diabéticos: estudo transversal no nordeste brasileiro. Revista Brasileira em Promoção da Saúde, 31(4), 2018, 1-10. 
Research, Society and Development, v. 10, n. 3, e31210313312, 2021

(CC BY 4.0) | ISSN 2525-3409 | DOI: http://dx.doi.org/10.33448/rsd-v10i3.13312

Zeni, A. L. B., Parisotto, A. V., Mattos, G., \& Helena, E. T. D. S. (2017). Utilização de plantas medicinais como remédio caseiro na Atenção Primária em Blumenau, Santa Catarina, Brasil. Ciência \& Saúde Coletiva, 22(8), 2703-2712. 